\title{
Molecular and Clinicopathological Features of Gastrointestinal Stromal Tumors in Vietnamese Patients
}

\author{
Quoc Dat Ngo, Quoc Thang Pham, Dang Anh Thu Phan, Anh Vu Hoang ${ }^{1}$, Thi Ngoc Ha Hua, Sao Trung Nguyen \\ Department of Pathology, ${ }^{1}$ Center for Molecular Biomedicine, University of Medicine and Pharmacy at Ho Chi Minh City, Ho Chi Minh City, Vietnam
}

\begin{abstract}
Background: Gastrointestinal stromal tumors (GISTs) are the most frequent mesenchymal neoplasms of the gastrointestinal tract. Management of GIST patients is currently based on clinicopathological features and associated genetic changes. However, the detailed characteristics and molecular genetic features of GISTs have not yet been described in the Vietnamese population. Methods: We first identified 155 patients with primary GIST who underwent surgery with primary curative intent between 2011 and 2014 at University Medical Center at Ho Chi Minh City, Vietnam. We evaluated the clinicopathological features and immunohistochemical reactivity to p53 and Ki-67 in these patients. Additionally, KIT genotyping was performed in 100 cases. Results: The largest proportion of GISTs was classified as high-risk (43.2\%). Of the 155 GISTs, 52 (33.5\%) were positive for Ki-67, and 58 (37.4\%) were positive for p53. The expression of Ki-67 and p53 were correlated with mitotic rate, tumor size, risk assessment, and tumor stage. Out of 100 GIST cases, KIT mutation was found in $68 \%$, of which $62(91.2 \%)$ were found in exon 11 , two $(2.9 \%)$ in exon 9 , and four $(5.8 \%)$ in exon 17 . No mutation in exon 13 was identified. Additionally, KIT mutations did not correlate with any clinicopathological features. Conclusions: The expression of $\mathrm{Ki}-67$ and p53 were associated with high-risk tumors. Mutations in exon 11 were the most commonly found, followed by exon 17 and exon 9. Additionally, KIT mutation status was not correlated with any recognized clinicopathological features.
\end{abstract}

Key Words: Gastrointestinal stromal tumors; Ki-67; p53; KIT mutation

Received: April 23, 2019 Revised: July 7, 2019 Accepted: August 27, 2019

Corresponding Author: Quoc Dat Ngo, MD, PhD, Department of Pathology, University of Medicine and Pharmacy at Ho Chi Minh City, 217 Hong Bang Street, Ward 11, District 5, Ho Chi Minh City, Vietnam

Tel: +84-838-558-411, Fax: +84-838-552-304, E-mail: ngoquocdat@ump.edu.vn

Gastrointestinal stromal tumors (GISTs) are the most frequent mesenchymal neoplasms of the gastrointestinal (GI) tract, ${ }^{1}$ with an incidence reported at $10-15$ per million persons. ${ }^{2}$ GISTs can be found anywhere within the GI tract, most commonly in the stomach $(55.6 \%)$ and small intestine $(31.8 \%)$. ${ }^{1,2}$ The cell of origin and diagnostic criteria were highly debated until gain-of-function mutations in KIT were confirmed in 1998. ${ }^{3-5}$ GISTs have a wide morphological spectrum, and although spindle cell tumors are the most common, $20 \%-25 \%$ of GIST cases present an epithelioid pattern, and some cases show mixed histology. Currently, diagnosis is based on morphology, ancillary tests including immunohistochemistry (95\% express CD117 and 98\% express DOG1), and molecular profiling. ${ }^{6-10}$ Among clinicopathological features, the most universally applicable prognostic factors for GISTs are tumor size and mitotic rate per 50 high power fields (HPF), ${ }^{1,7}$,

Nearly $80 \%$ of GISTs harbor a mutation in KIT, and another
$5 \%-10 \%$ of cases have platelet-derived growth factor receptor (PDGFR) mutation. ${ }^{6}$ However, approximately $10 \%-15 \%$ of GISTs have no mutation in either of these genes and were previously known as wild-type GISTs. ${ }^{6}$ However, the terminology "wild-type GISTs" is progressively being abandoned as mutations have been identified in BRAF and succinate dehydrogenase $(S D H)$ genes. ${ }^{910}$ In tumors with a KIT mutation, the most commonly affected region is exon 11 (juxtamembrane domain; 70\%), followed by exon 9 (extracellular domain; 10\%-15\%), exon 13 (tyrosine kinase 1 domain; 1\%-2\%), and exon 17 (activation loop; $1 \%){ }^{11-14}$ Secondary mutations in exons 13,14 , or 17 are usually present in imatinib-resistant patients. ${ }^{15}$

Management of GIST patients is currently based not only on clinicopathological features (e.g., risk assessment and tumor stage), but also genetic changes including KIT-, PDGFRA-, and SDH mutations. ${ }^{8}$ To our knowledge, however, the detailed characteristics and molecular genetic features of GISTs have not yet been 
described in the Vietnamese population.

In this study, we conducted a standard clinicopathological risk assessment and evaluated the relationships between expression of $\mathrm{Ki}-67$ and p53 and the clinicopathological features of GISTs in Vietnamese patients. Furthermore, we investigated the common regions of KIT mutation, comprising exon 11, exon 9, exon 13 , and exon 17.

\section{MATERIALS AND METHODS}

\section{Tissue samples}

In a descriptive study design, 155 primary GISTs were collected from patients who underwent surgical resection between 2011 and 2014 at the University Medical Center at Ho Chi Minh City (Ho Chi Minh City, Vietnam). Patients treated with preoperative imatinib were not enrolled in the study. The diagnosis was confirmed based on the histological features and immunoreactivity of CD117.

Clinicopathological data of age, sex, tumor location, tumor size, and tumor stage were recorded. Localized GISTs were those that were confined to the primary organ of origin, and locally advanced GISTs were those with contiguous organ involvement. Morphological features, such as pattern (spindle cell, epithelioid, or mixed) and mitotic activity (per $50 \mathrm{HPFs}$ with a total area of $5 \mathrm{~mm}^{2}$ ) were evaluated. For risk assessment, tumors were categorized into very low-, low-, intermediate-, and high-risk groups based on the National Institute of Health (NIH) classification criteria. $^{16}$

For immunohistochemical analysis, we used archival formalin-fixed, paraffin-embedded (FFPE) tissues from the 155 GISTs. One or two representative tumor blocks from each case were examined using immunohistochemistry.

For KIT genotyping, 100 FFPE tissues were available for KIT mutation analysis. Genomic DNA was isolated from paraffinembedded tissues and tested at KIT exons 9, 11, 13, and 17.

\section{Immunohistochemistry}

Three-micrometer-thick slides were used for immunohistochemical analysis. Primary antibodies for CD117 (1:400, polyclonal, Ventana, Oro Valley, AZ, USA), Ki-67 (1:25, MIB-1 monoclonal, Dako, Carpinteria, CA, USA), p53 (1:50, DO-7 monoclonal, Dako), smooth muscle actin (SMA; 1:2,000, 1A4, monoclonal, Dako), and neuron-specific enolase (NSE; 1:50, VI-H14, Dako) were used to detect protein expression. Immunostaining for CD117, Ki-67, p53, SMA, and NSE was performed using an automated staining machine (VENTANA
BenchMark, Ventana) according to the manufacturer's instructions. For the markers (CD117, SMA, and NSE), immunostaining was defined as positive if $\geq 10 \%$ of tumor cells were stained and negative if $<10 \%$ of tumor cells were stained. The labeling index (LI) (\%) for Ki-67 and p53 expression was evaluated based on a count of at least 1,000 tumor cells in the highest-density immunoreactivity areas of the positive tumor nuclei. Ki-67 and p53 were defined as positive if the LI was higher than $10 \%{ }^{17}$ or $5 \%,{ }^{18}$ respectively. Two senior surgical pathologists without knowledge of the clinicopathological features of the patients independently reviewed the immunostaining slides. Interobserver differences were resolved by consensus review using a doubleheaded microscope after independent review.

\section{KIT genotyping}

Genomic DNA was extracted from paraffin-embedded tissues of 100 patients using the ReliaPrep FFPE gDNA Miniprep System kit (Promega, Madison, WI, USA) according to the manufacturer's protocol. Amplifications of KIT exons 9, 11, 13, and 17 were performed using TaKaRa Taq HotStart Polymerase (Takara Bio, Shiga, Japan) with primers as previously published. ${ }^{19}$ Polymerase chain reaction (PCR) fragments were sequenced and analyzed in both sense and antisense directions using a BigDye Terminator v3.1 kit on an ABI 3130 Genetic Analyzer (Applied Biosystems, Foster City, CA, USA). Mutations were confirmed through a second independent PCR amplification and sequencing.

\section{Statistical analysis}

Associations between clinicopathological features and $\mathrm{Ki}-67$ expression, p53 expression, and KIT mutations were analyzed using the chi-square test and Fisher's exact test. Differences were considered significant at $\mathrm{p}<.05$. All statistical analyses were performed using SPSS software ver. 16.0 (SPSS Inc., IBM, Chicago, IL, USA).

\section{Ethics statement}

This study was approved by the Board of Ethics in Biomedical Research at the University of Medicine and Pharmacy at Ho Chi Minh City, Vietnam (approval number: 54/UMP-BOARD; Date: December 22, 2012). Informed consent was obtained from all individual participants included in the study. 


\section{RESULTS}

\section{Clinicopathological characteristics of GISTs}

We included $72(46.5 \%)$ male and $83(53.5 \%)$ female patients in this study, with a male: female ratio of 1:1.2, and the median age at diagnosis was 55 years (range, 15 to 88 years). Tumor size ranged from 1.0 to $29 \mathrm{~cm}$ in the largest dimension, with a median size of $6 \mathrm{~cm}$. The GISTs presented in a wide distribution both within and outside the GI tract (Table 1). The most common location was the stomach $(52.3 \%)$, followed by the jejunum-ileum $(27.7 \%)$. GISTs were also found outside the GI tract including the omentum $(5.8 \%)$ and retroperitoneum $(3.2 \%)$.

Most cases showed spindle cell $(72.3 \%)$ or epithelioid cell morphology (14.8\%), and mixed type accounted for $12.9 \%$ of cases (Table 1, Fig. 1A-C). Tumor cells were positive for CD117 (Fig. 1D). Many GISTs were positive for SMA (20\%) (Fig. 1E) or for NSE $(67.7 \%)$ (Fig. 1F). Sixty-seven of 155 cases (43.2\%) were classified as high risk, $42(27.1 \%)$ as low risk, $40(25.8 \%)$ as intermediate risk, and $6(3.9 \%)$ as very low risk (Supplementary Table S1). Most tumors located in the jejunum-ileum (55.8\%) or the colorectum $(44.4 \%)$ fell into the high-risk category (Table 2). Of the 14 cases of extra-intestinal GISTs, 13 (92.9\%) were classified as the high-risk group.

We next evaluated Ki-67 and p53 expression in the GISTs (Fig. 1G, H). Of the 155 cases, $52(33.5 \%)$ were positive for

Table 1. Characteristics of 155 patients with GISTs

\begin{tabular}{lc}
\hline Characteristic & No. $(\%)(\mathrm{n}=155)$ \\
\hline Age, median (yr) & $55(15-88)$ \\
Sex & \\
Female & $83(53.5)$ \\
Male & $72(46.5)$ \\
Tumor location & \\
Stomach & $81(52.3)$ \\
Duodenum & $8(5.2)$ \\
Jejunum-ileum & $43(27.7)$ \\
Colorectum & $9(5.8)$ \\
Omentum & $9(5.8)$ \\
Retroperitoneum & $5(3.2)$ \\
Histology & \\
Spindle & $112(72.3)$ \\
Epithelioid & $23(14.8)$ \\
Mixed & $20(12.9)$ \\
Immunohistochemical profiles & \\
SMA & $31(20.0)$ \\
NSE & $105(67.7)$ \\
Ki-67 & $52(33.5)$ \\
p53 & $58(37.4)$ \\
\hline
\end{tabular}

GISTs, gastrointestinal stromal tumors; SMA, smooth muscle actin; NSE, neuron-specific enolase.
Ki-67, and $58(37.4 \%)$ were positive for p53 (Table 1). Expression of Ki-67 correlated with mitotic rate $(\mathrm{p}<.001)$, tumor size $(\mathrm{p}=.014)$, risk assessment $(\mathrm{p}<.001)$, and tumor stage $(\mathrm{p}<.001)$ (Table 3). Similarly, expression of p53 correlated with mitotic rate $(\mathrm{p}<.001)$, tumor risk assessment $(\mathrm{p}<.001)$, and tumor stage $(\mathrm{p}<.001)$. In contrast, expression of $\mathrm{Ki}-67$ and p53 was not associated with GIST phenotype $(\mathrm{p}=.482$ and $\mathrm{p}=.102$, respectively).

\section{KIT mutation analysis}

KIT mutations were detected in 68 of 100 GIST cases $(68 \%)$. Sixty-two of the 68 KIT mutations $(91.2 \%)$ were found in KIT exon 11 (Table 4). There were two cases $(2.9 \%)$ with mutations in KIT exon 9 and 4 cases $(5.8 \%)$ with mutations in KIT exon 17. No mutations were found in KIT exon 13 . We detected a variety of KIT mutation types in exons 9, 11, and 17 including point mutations, insertions, deletions, duplications, and complex mutations.

Among the cases with mutations in KIT exon 11, in-frame deletion was the most common type $(35 / 62,56.5 \%)$ (Fig. 2A), followed by point mutation $(19 / 62,30.6 \%$ ) (Fig. 2B). Complex mutations were detected in six of 62 cases $(9.7 \%)$. The two cases with mutation in the extracellular membrane region encoded by exon 9 were insertions (Y503_F504insAY) (Fig. 2C) and were found in tumors of the jejunum-ileum. All mutations in KIT exon 17 were point mutations, and three of four cases $(75 \%)$ had an N822K substitution (Fig. 2D). We further investigated the relationships between KIT mutation status and other clinicopathological characteristics; however, no significant correlation was found (Supplementary Table S2).

\section{DISCUSSION}

A systematic review of 29 studies on nearly 14,000 GIST patients reported a median age of 60 years, and the incidence was similar for males and females. ${ }^{2}$ Our study showed a slightly higher incidence rate in females and a median age lower than in other studies. However, our average age was higher than that of the Korean sub-population reported in the systemic review mentioned above. ${ }^{2}$ Although many studies have reported the presence of GISTs in the esophagus, ${ }^{1,2}$ no esophageal GISTs were present in our study. Our results showed that GISTs were most predominant in the stomach, followed by the small intestine, which was consistent with the findings in most of the literature. Interestingly, our results revealed that extra-intestinal GISTs accounted for $9 \%$ of cases. According to a study in Singapore from 

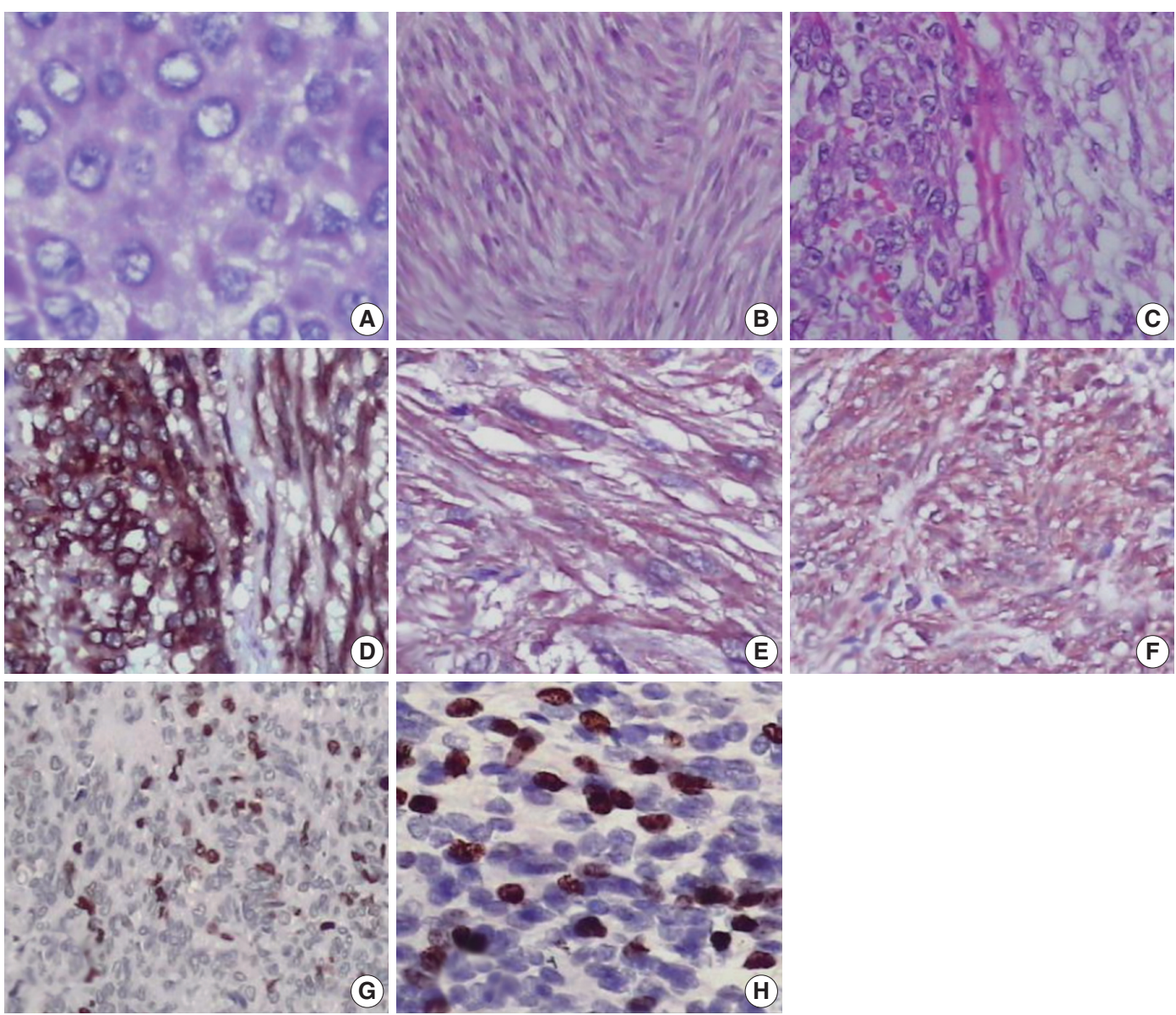

Fig. 1. Histopathological and immunohistochemical analysis of gastrointestinal stromal tumor. (A) Epithelioid cell morphology. (B) Spindle cell morphology. (C) Mixed type. Immunohistochemical expression of CD117 (D), smooth muscle actin (E), neuron-specific enolase (F), p53 (G), and Ki-67 (H).

Table 2. Location and risk stratification of 155 GISTs

\begin{tabular}{llrrr}
\hline & Very low risk & Low risk & Intermediate risk & High risk \\
\hline Stomach & $6(7.4)$ & $30(37.0)$ & $22(27.2)$ & $23(28.4)$ \\
Duodenum & 0 & $1(12.5)$ & $4(50.0)$ & $3(37.5)$ \\
Jejunum-ileum & 0 & $9(20.9)$ & $10(23.3)$ & $24(55.8)$ \\
Colorectum & 0 & $1(11.1)$ & $4(44.4)$ & $4(44.4)$ \\
Omentum & 0 & $1(11.1)$ & 0 & $8(88.9)$ \\
Retroperitoneum & 0 & 0 & 0 & $5(100)$ \\
\hline
\end{tabular}

Values are presented as number (\%).

GISTs, gastrointestinal stromal tumors.

1998 to 2008, the frequency of extra-intestinal GISTs has increased since CD117 immunohistochemistry has been applied for diagnosis. ${ }^{20}$

According to the literature, most GISTs have high-risk features, followed by intermediate- and low-risk groups. ${ }^{2,17,18,20,21}$ Our study also had a majority of GISTs classified as high-risk, followed by low-risk, intermediate-, and very low-risk groups. Our study revealed that most jejunum-ileum and colorectal GISTs fell into the high-risk group. These results were compatible with a previous study in which non-gastric GISTs were mostly classified in the high-risk group. ${ }^{22}$ Our study showed an extremely high ratio of extra-intestinal GISTs in the high-risk group. In contrast, another study with 29 extra-intestinal GIST cases showed seven cases $(24.1 \%)$ in the high-risk group using the criteria of mitotic rate per 50 high power fields $\geq 5 / 50 \mathrm{HPF}$ and $\mathrm{Ki}-67 \geq 10 \% .{ }^{23}$ Recently reported evidence proposed that retroperitoneal GISTs could be derived from a GI tract origin. ${ }^{24}$

In our study, Ki-67 expression correlated with risk assessment and other clinicopathological features, as in previous studies. ${ }^{17,18,25,26}$ Although the cut-off value for Ki-67 expression varied among studies, it is clear that Ki-67 expression is a prognostic factor and a good marker for biological behavior such as metastatic tendency. ${ }^{17,18,25,26}$ Furthermore, TP53, the most mutated gene in human cancer, ${ }^{27}$ has also been proposed to be a predictive 
Table 3. Relationship between Ki-67 expression, p53 expression and clinicopathological characteristics of GISTs

\begin{tabular}{|c|c|c|c|c|c|c|}
\hline & \multicolumn{3}{|c|}{ Ki-67 expression } & \multicolumn{3}{|c|}{ p53 expression } \\
\hline & Positive & Negative & $\mathrm{p}$-value & Positive & Negative & $p$-value \\
\hline Mitotic rate (per 50 HPFs) & & & $<.001^{a}$ & & & $.001^{a}$ \\
\hline$\leq 5$ & 19 (20.2) & 75 (79.8) & & 25 (26.6) & $69(73.4)$ & \\
\hline $6-10$ & $5(25.0)$ & $15(75.0)$ & & $7(35.0)$ & $13(65.0)$ & \\
\hline$>10$ & $28(68.3)$ & $13(31.7)$ & & $26(63.4)$ & $15(36.6)$ & \\
\hline Tumor size (cm) & & & $.014^{a}$ & & & $.148^{a}$ \\
\hline $0-5$ & $16(24.2)$ & $50(75.8)$ & & $20(30.3)$ & $46(69.7)$ & \\
\hline$>5-10$ & $17(32.1)$ & $36(67.9)$ & & $20(37.7)$ & $33(62.3)$ & \\
\hline$>10$ & $19(52.8)$ & 17 (47.2) & & $18(50.0)$ & $18(50.0)$ & \\
\hline Risk stratification & & & $<.001^{\mathrm{a}}$ & & & $<.001^{\mathrm{a}}$ \\
\hline Very low/low risk & $6(12.5)$ & $42(87.5)$ & & $8(16.7)$ & $40(83.3)$ & \\
\hline Intermediate risk & $10(25.0)$ & $30(75.0)$ & & $15(37.5)$ & $25(62.5)$ & \\
\hline High risk & $36(53.7)$ & 31 (46.3) & & $35(52.2)$ & $32(47.8)$ & \\
\hline Histology & & & $.482^{\mathrm{a}}$ & & & $.102^{\mathrm{a}}$ \\
\hline Spindle & $77(68.8)$ & 35 (31.2) & & $75(67.0)$ & $37(33.0)$ & \\
\hline Epithelioid & $15(65.2)$ & $8(34.8)$ & & $10(43.5)$ & $13(56.5)$ & \\
\hline Mixed & $11(55.0)$ & $9(45.0)$ & & $12(60.0)$ & $8(40.0)$ & \\
\hline Tumor necrosis & & & $.475^{b}$ & & & $.012^{\mathrm{b}}$ \\
\hline No & 32 (31.4) & 70 (68.6) & & $31(30.4)$ & 71 (69.6) & \\
\hline Yes & $20(37.7)$ & 33 (62.3) & & 27 (50.9) & $26(49.1)$ & \\
\hline Stage $^{c}$ & & & $<.001^{b}$ & & & $<.001^{\mathrm{b}}$ \\
\hline Localized & $12(17.4)$ & 57 (82.6) & & 14 (20.3) & 55 (79.7) & \\
\hline Locally advanced & 29 (50.9) & $28(49.1)$ & & 30 (52.6) & $27(47.4)$ & \\
\hline
\end{tabular}

Values are presented as number (\%).

GISTs, gastrointestinal stromal tumors; HPFs, high-power fields.

${ }^{a}$ Chi-square test; ${ }^{b}$ Fisher's exact test; ' 29 GISTs patients were excluded from the analysis as they were missing tumor stage information.

Table 4. Summary of KIT mutation status in 100 GIST patients

\begin{tabular}{|c|c|c|c|c|c|}
\hline & \multicolumn{4}{|c|}{ KIT mutation } & \multirow{2}{*}{$\begin{array}{c}K I T \\
\text { wild-type }\end{array}$} \\
\hline & Exon 9 & Exon 11 & Exon 13 & Exon 17 & \\
\hline \multicolumn{6}{|l|}{ Tumor location } \\
\hline Stomach & 0 & 39 & 0 & 2 & 14 \\
\hline Duodenum & 0 & 3 & 0 & 0 & 1 \\
\hline Jejunum-ileum & 2 & 14 & 0 & 1 & 7 \\
\hline Colorectum & 0 & 1 & 0 & 0 & 6 \\
\hline Omentum & 0 & 3 & 0 & 1 & 3 \\
\hline Retroperitoneum & 0 & 2 & 0 & 0 & 1 \\
\hline \multicolumn{6}{|l|}{ Type of mutation } \\
\hline In-frame deletion & 0 & 35 & 0 & 0 & 0 \\
\hline Single point & 0 & 19 & 0 & 4 & 0 \\
\hline Complex & 0 & 6 & 0 & 0 & 0 \\
\hline Insertion & 2 & 1 & 0 & 0 & 0 \\
\hline Duplication & 0 & 1 & 0 & 0 & 0 \\
\hline
\end{tabular}

GIST, gastrointestinal stromal tumor.

marker for risk of malignancy in GISTs. ${ }^{17,28}$ The cut-off value for p53 expression varied among studies. ${ }^{17,18,28}$ One study from Japan that used the same cut-off values for $\mathrm{p} 53$ expression and risk assessment as our study showed less frequent $\mathrm{p} 53$ expression in the high-risk group. ${ }^{18}$ However, in our study, there was a significant correlation between $\mathrm{p} 53$ expression and high-risk assessment.
Pauser et al. ${ }^{28}$ reported that expression of $\mathrm{p} 53$ was related to epithelioid morphology. However, our study showed that p 53 expression was not correlated with the morphologic phenotypes of GISTs.

In the current study, KIT mutations were found in 60\%-80\% of GISTs and were most frequently detected in exon 11, which is consistent with previous reports. ${ }^{14,6,29,30}$ Additionally, KIT mutations were not associated with the clinicopathological features of GISTs. According to the National Comprehensive Cancer Network (NCCN) panel, the presence and type of KIT mutation are not strongly correlated with prognosis. ${ }^{8}$ However, another study showed that GISTs with KIT exon 11 deletions and high mitotic rates were negatively correlated with recurrencefree survival. ${ }^{31}$ In the American College of Surgeons Oncology Group (ACOSOG) Z9001 trial, GIST patients with KIT exon 11 deletion mutations had longer recurrence-free survival than those with other mutation types (genotypes) when treated with adjuvant imatinib. ${ }^{32}$

Interestingly, the second most frequent region of KIT mutation in this study was exon 17. Two of four cases with KIT exon 17 mutations were identified in the stomach. Other studies have reported that primary mutations in KIT exon 17 were fre- 

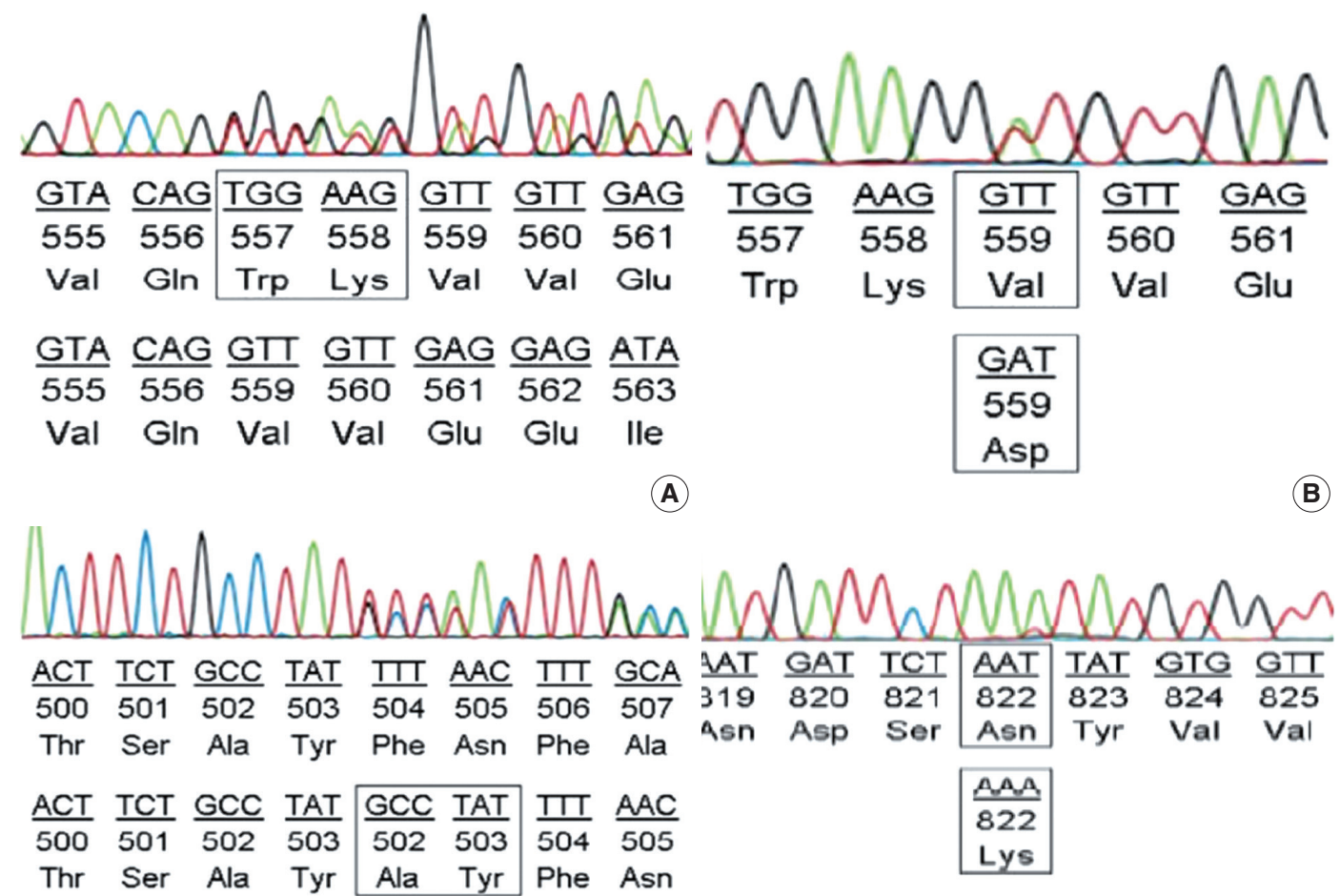

Fig. 2. KIT mutation in gastrointestinal stromal tumor. (A) In-frame deletion in exon 11. (B) Point mutation in exon 11. (C) Insertion mutation in exon 9. (D) Point mutation in exon 17.

quently detected in tumors of the small intestine, ${ }^{11,33}$ and approximately $1 \%$ of newly diagnosed GISTs had detectable mutations in KIT exon 17. ${ }^{11}$ Furthermore, KIT exon 17 mutations as a secondary mutation (including N822K substitution) were often found in cases with acquired imatinib resistance. ${ }^{34,35}$ Our study did not detect any mutations in KIT exon 13, and mutations in KIT exon 9 were only identified in two cases. According to the NCCN guidelines, patients with advanced GISTs with KIT exon 9 mutations who are treated with double the standard dose of imatinib have an improved likelihood of response. ${ }^{8}$

Our study has several limitations. Because it is a descriptive study, it lacks clinical outcome information for the patients, which would enable us to determine overall and disease-free survival. Furthermore, additional analysis to investigate the status of $P D G$ FRA and SDH mutations in the "wild-type" GIST cases (32\%) to reveal a more comprehensive picture of the molecular alterations in Vietnamese GIST patients would be helpful but was not feasible at this point.

In conclusion, this study demonstrated the clinicopathological features, immunohistochemical characteristics, and KIT mutation status of 155 Vietnamese GIST patients. The stomach was the most common site of GISTs, followed by the small intestine, outside the GI tract, and colorectum. The majority of GISTs outside the GI tract fell into the high-risk group. Expression of Ki67 and p53 was associated with high-risk assessment. Mutations in KIT exon 11 were the most frequently detected, followed by mutations in KIT exons 17 and 9. However, KIT mutations were not associated with clinicopathological or morphological features.

\section{Electronic Supplementary Material}

Supplementary materials are available at Journal of Pathology and Translational Medicine (https://jpatholtm.org).

\section{ORCID}

Quoc Dat Ngo: https://orcid.org/0000-0003-1461-0216

Quoc Thang Pham:: https://orcid.org/0000-0001-8787-367X

Dang Anh Thu Phan: https://orcid.org/0000-0002-4062-0904

Sao Trung Nguyen: https:/orcid.org/0000-0002-3847-4717

\section{Author Contributions}

Conceptualization: QDN.

Data curation: QDN, QTP, AVH. 
Formal analysis: QDN, QTP.

Investigation: QDN.

Methodology: QDN, QTP, DATP.

Project administration: QDN, TNHH, STN.

Resources: QDN.

Supervision: TNHH, STN.

Validation: QDN, TNHH, STN.

Visualization: QDN, QTP.

Writing —original draft: QDN, QTP, DATP.

Writing—review \& editing: QDN, QTP, DATP.

\section{Conflicts of Interest}

The authors declare that they have no potential conflicts of interest.

\section{Funding}

No funding to declare.

\section{Acknowledgments}

We thank Professor Lewis Hassell, Department of Pathology, Oklahoma University Health Sciences Center for English proofreading.

\section{REFERENCES}

1. Miettinen M, Fletcher CD, Kindblom LG, Tsui WM. Mesenchymal tumours of the stomach. In: Bosman FT, Carneiro F, Hruban RH, Theise ND, eds. WHO classification of tumours of the digestive system. 3rd ed. Lyon: IARC, 2010; 74-9.

2. Søreide K, Sandvik OM, Søreide JA, Giljaca V, Jureckova A, Bulusu VR. Global epidemiology of gastrointestinal stromal tumours (GIST): a systematic review of population-based cohort studies. Cancer Epidemiol 2016; 40: 39-46.

3. Mazur MT, Clark HB. Gastric stromal tumors: reappraisal of histogenesis. Am J Surg Pathol 1983; 7: 507-19.

4. Hirota S, Isozaki K, Moriyama Y, et al. Gain-of-function mutations of c-kit in human gastrointestinal stromal tumors. Science 1998; 279: 577-80.

5. Joensuu H. Gastrointestinal stromal tumor (GIST). Ann Oncol 2006; 17 Suppl 10: x280-6.

6. Von Mehren M, Randall RL, Benjamin RS, et al. Gastrointestinal stromal tumors, version 2.2014. J Natl Compr Canc Netw 2014; 12: 853-62.

7. Miettinen M, Lasota J. Gastrointestinal stromal tumors. Gastroenterol Clin North Am 2013; 42: 399-415.

8. Soft tissue sarcoma, version 1.2018. National Comprehensive Cancer
Network: Clinical Practice Guidelines in Oncology [Internet]. Plymouth Meeting: National Comprehensive Cancer Network, 2018 [cited 2017 Dec 14]. Available from: https://www.nccn.org/ professionals/physician_gls/pdf/sarcoma.pdf.

9. Agaram NP, Wong GC, Guo T, et al. Novel V600E BRAF mutations in imatinib-naive and imatinib-resistant gastrointestinal stromal tumors. Genes Chromosomes Cancer 2008; 47: 853-9.

10. Gaal J, Stratakis CA, Carney JA, et al. SDHB immunohistochemistry: a useful tool in the diagnosis of Carney-Stratakis and Carney triad gastrointestinal stromal tumors. Mod Pathol 2011; 24: 147-51.

11. Patrikidou A, Domont J, Chabaud S, et al. Long-term outcome of molecular subgroups of GIST patients treated with standard-dose imatinib in the BFR14 trial of the French Sarcoma Group. Eur J Cancer 2016; 52: 173-80.

12. Heinrich MC, Owzar K, Corless CL, et al. Correlation of kinase genotype and clinical outcome in the North American Intergroup Phase III Trial of imatinib mesylate for treatment of advanced gastrointestinal stromal tumor: CALGB 150105 Study by Cancer and Leukemia Group B and Southwest Oncology Group. J Clin Oncol 2008; 26: 5360-7.

13. Lasota J, Miettinen M. Clinical significance of oncogenic KIT and PDGFRA mutations in gastrointestinal stromal tumours. Histopathology 2008; 53: 245-66.

14. Heinrich MC, Rankin C, Blanke CD, et al. Correlation of long-term results of imatinib in advanced gastrointestinal stromal tumors with next-generation sequencing results: analysis of phase 3 SWOG intergroup trial S0033. JAMA Oncol 2017; 3: 944-52.

15. Heinrich MC, Maki RG, Corless CL, et al. Primary and secondary kinase genotypes correlate with the biological and clinical activity of sunitinib in imatinib-resistant gastrointestinal stromal tumor. J Clin Oncol 2008; 26: 5352-9.

16. Joensuu H. Risk stratification of patients diagnosed with gastrointestinal stromal tumor. Hum Pathol 2008; 39: 1411-9.

17. Hu TH, Chuah SK, Lin JW, et al. Expression and prognostic role of molecular markers in 99 KIT-positive gastric stromal tumors in Taiwanese. World J Gastroenterol 2006; 12: 595-602.

18. Nakamura N, Yamamoto H, Yao T, et al. Prognostic significance of expressions of cell-cycle regulatory proteins in gastrointestinal stromal tumor and the relevance of the risk grade. Hum Pathol 2005; 36: 828-37.

19. Vu HA, Xinh PT, Kikushima M, et al. A recurrent duodenal gastrointestinal stromal tumor with a frameshift mutation resulting in a stop codon in KIT exon 13. Genes Chromosomes Cancer 2005; 42: 179-83.

20. Chiang NJ, Chen LT, Tsai CR, Chang JS. The epidemiology of gastrointestinal stromal tumors in Taiwan, 1998-2008: a nation-wide 
cancer registry-based study. BMC Cancer 2014; 14: 102.

21. Brabec P, Sufliarsky J, Linke Z, et al. A whole population study of gastrointestinal stromal tumors in the Czech Republic and Slovakia. Neoplasma 2009; 56: 459-64.

22. Tryggvason G, Gíslason HG, Magnússon MK, Jónasson JG. Gastrointestinal stromal tumors in Iceland, 1990-2003: the icelandic GIST study, a population-based incidence and pathologic risk stratification study. Int J Cancer 2005; 117: 289-93.

23. Yamamoto H, Oda Y, Kawaguchi K, et al. c-kit and PDGFRA mutations in extragastrointestinal stromal tumor (gastrointestinal stromal tumor of the soft tissue). Am J Surg Pathol 2004; 28: 479-88.

24. Miettinen M, Felisiak-Golabek A, Wang Z, Inaguma S, Lasota J. GIST manifesting as a retroperitoneal tumor: clinicopathologic immunohistochemical, and molecular genetic study of 112 cases. Am J Surg Pathol 2017; 41: 577-85.

25. Belev B, Brčić I, Prejac J, et al. Role of Ki-67 as a prognostic factor in gastrointestinal stromal tumors. World J Gastroenterol 2013; 19: 523-7.

26. Ohdaira H, Ohyama S, Yamaguchi T, Yanagisawa A, Kato Y, Urashima M. Ki67 and tumor size as prognostic factors of gastrointestinal stromal tumors. Japan Med Assoc J 2005; 48: 586-92.

27. Kandoth C, McLellan MD, Vandin F, et al. Mutational landscape and significance across 12 major cancer types. Nature 2013; 502: 333-9.

28. Pauser U, Schmedt Auf der Günne N, Klöppel G, Merz H, Feller AC. P53 expression is significantly correlated with high risk of malignancy and epithelioid differentiation in GISTs: an immuno- histochemical study of 104 cases. BMC Cancer 2008; 8: 204.

29. Corless CL, Heinrich MC. Molecular pathobiology of gastrointestinal stromal sarcomas. Annu Rev Pathol 2008; 3: 557-86.

30. Tzen CY, Wang MN, Mau BL. Spectrum and prognostication of KIT and PDGFRA mutation in gastrointestinal stromal tumors. Eur J Surg Oncol 2008; 34: 563-8.

31. Joensuu H, Wardelmann E, Sihto $\mathrm{H}$, et al. Effect of KIT and PDGFRA mutations on survival in patients with gastrointestinal stromal tumors treated with adjuvant imatinib: an exploratory analysis of a randomized clinical trial. JAMA Oncol 2017; 3: 602-9.

32. Corless CL, Ballman KV, Antonescu CR, et al. Pathologic and molecular features correlate with long-term outcome after adjuvant therapy of resected primary GI stromal tumor: the ACOSOG Z9001 trial. J Clin Oncol 2014; 32: 1563-70.

33. Lasota J, Corless CL, Heinrich MC, et al. Clinicopathologic profile of gastrointestinal stromal tumors (GISTs) with primary KIT exon 13 or exon 17 mutations: a multicenter study on 54 cases. Mod Pathol 2008; 21: 476-84.

34. Garner AP, Gozgit JM, Anjum R, et al. Ponatinib inhibits polyclonal drug-resistant KIT oncoproteins and shows therapeutic potential in heavily pretreated gastrointestinal stromal tumor (GIST) patients. Clin Cancer Res 2014; 20: 5745-55.

35. Gao J, Tian Y, Li J, Sun N, Yuan J, Shen L. Secondary mutations of c-KIT contribute to acquired resistance to imatinib and decrease efficacy of sunitinib in Chinese patients with gastrointestinal stromal tumors. Med Oncol 2013; 30: 522. 